\title{
Partial Molar Entropy and Partial Molar Heat Capacity of Electrons in Metals and Superconductors
}

\author{
Alan L. Rockwood ${ }^{1,2}$ \\ ${ }^{1}$ Department of Pathology, University of Utah School of Medicine, Salt Lake City, UT, USA \\ ${ }^{2}$ Medical Directors Department, ARUP Laboratories, Salt Lake City, UT, USA \\ Email: alan.rockwood@aruplab.com
}

Received 9 November 2015; accepted 26 January 2016; published 29 January 2016

Copyright (C) 2016 by author and Scientific Research Publishing Inc. This work is licensed under the Creative Commons Attribution International License (CC BY). http://creativecommons.org/licenses/by/4.0/

\section{(c) (i) Open Access}

\section{Abstract}

There are at least two valid approaches to the thermodynamics of electrons in metals. One takes a microscopic view, based on models of electrons in metals and superconductor and uses statistical mechanics to calculate the total thermodynamic functions for the model-based system. Another uses partial molar quantities, which is a rigorous thermodynamic method to analyze systems with components that can cross phase boundaries and is particularly useful when applied to a system composed of interacting components. Partial molar quantities have not been widely used in the field of solid state physics. The present paper will explore the application of partial molar electronic entropy and partial molar electronic heat capacity to electrons in metals and superconductors. This provides information that is complementary information from other approaches to the thermodynamics of electrons in metals and superconductors and can provide additional insight into the properties of those materials. Furthermore, the application of partial molar quantities to electrons in metals and superconductors has direct relevance to long-standing problems in other fields, such as the thermodynamics of ions in solution and the thermodynamics of biological energy transformations. A unifying principle between reversible and irreversible thermodynamics is also discussed, including how this relates to the completeness of thermodynamic theory.

\section{Keywords}

Partial Molar Entropy, Partial Molar Heat Capacity, Electronic Entropy, Electronic Heat Capacity, Partial Molar Electronic Entropy, Partial Molar Electronic Heat Capacity 


\section{Introduction}

Entropy and heat capacity measurements have long played an important role in providing insight into the electronic structure of metals and superconductors [1] [2]. There are at least two valid approaches to the thermodynamics of electrons in metals. One takes a microscopic view, based on models of electrons in metals and superconductor and uses statistical mechanics to calculate the total thermodynamic functions for the model-based system. The simplest model is the Sommerfeld model, which is based on the properties of a degenerate free electron gas. Beyond this there is a hierarchy of increasingly sophisticated models of increasing realism, usually based on concepts from the band theory of solids. An important property of this approach is that it is entirely model-dependent. Furthermore, parsing of the entropy between interacting components of a system (e.g. electrons interacting with the lattice of a metal) tends to be somewhat heuristic or even a bit arbitrary.

Another approach uses partial molar quantities. This is a rigorous thermodynamic method to treat systems with components that can cross phase boundaries. It is widely used in classical chemical thermodynamics, especially in the analysis of the thermodynamics of solutions. However, partial molar methods can be applied to any system wherein components can be added to or removed from a phase (at least in infinitesimal amounts), which would therefore apply to metals and superconductors. Being a purely thermodynamic approach, it is based on macroscopic thermodynamic functions and does not depend on any microscopic models for its validity. However, it can sometimes be profitably applied to microscopic models to provide additional insight into the properties of systems under study. One of the features of the partial molar approach to thermodynamics is that it provides an unambiguous way to parse extensive thermodynamic functions, such as the entropy of a system of interacting components into contributions from each component.

\section{Partial Molar Quantities}

A partial molar quantity is based on the idea that an extensive thermodynamic variable may change upon the addition or removal of an infinitesimal amount of one of the components of the system. For example, if $d n_{i}$ is a small quantity of component $i$ added to a system then the partial molar entropy of that component of the system is given by

$$
\bar{S}_{i}=\left(\frac{\partial S}{\partial n_{i}}\right)_{T, P}
$$

where $\bar{S}_{i}$ is the partial molar entropy of component $i, S$ is the total entropy of the system, and the subscripts $T$ and $P$ denote constant temperature and external pressure. It is also to be understood that in Equation (1), and other equations in the paper unless otherwise stated, the composition of all of the components are held constant except for component $i$.

Among chemists partial molar quantities are normally specified in terms of moles, hence the word "molar" in the term "partial molar". (In some of the earlier literature these quantities have also been referred to as "partial molal" quantities.) However, in this paper it is sometimes more convenient to express partial molar quantities in terms of number of particles (e.g. electrons), and the partial molar quantities in the present paper are based on this view unless otherwise specified.

In general, partial molar quantities are useful for systems of variable compositions, systems in which components can cross phase boundaries, and systems composed of interacting components. Metals and superconductors possess all of these properties, given the fact that it is possible to add or remove infinitesimal numbers of electrons from a sample of metal or superconductor, electrons can be transferred across phase boundaries, and electrons interact strongly with the lattice.

One of the properties of partial molar quantities is that they provide a rigorous and systematic way to split an extensive thermodynamic function of a system containing interacting components into contributions from each component. For example, in solution phase chemistry the total entropy of an aqueous sucrose solution would be the sum of two terms:

$$
S=n_{1} \bar{S}_{1}+n_{2} \bar{S}_{2}
$$

where $n_{1}$ is the number of moles (or molecules) of water in the solution, $\bar{S}_{1}$ is the partial molar entropy of water in the solution, $n_{2}$ is the number of moles (or molecules) of sucrose in the solution, and $\bar{S}_{2}$ is the par- 
tial molar entropy of sucrose in the solution. The term $n_{1} \bar{S}_{1}$ is the contribution of component 1 to the total entropy, and $n_{2} \bar{S}_{2}$ is the contribution of component 2. Though a sucrose solution was used for purposes of illustration, this equation applies to any two-component system whose components can cross phase boundaries. An analogous equation can be extended to any number of components of a complex mixture, one term in the sum for each component [3].

By analogy to solution-phase thermodynamics, the entropy of a metal can be treated in terms of partial molar quantities, where $n_{1}$ in Equation (2) would be the number of electrons, $\bar{S}_{1}$ would be the partial molar entropy of electrons in the metal, $n_{2}$ would be the number of lattice elements (e.g. lattice ions or volume elements, depending on the material or model), and $\bar{S}_{2}$ would be the partial molar entropy of lattice elements. Similarly, the entropy of an alloy is treatable as a multi-component system by extending the sum to include additional terms representing the various components in the alloy, including the various types of lattice ions that may be present as well as conduction electrons.

The partial molar method of splitting a thermodynamic function into contributions from the components of a system implicitly takes into account any interactions between the system components. Furthermore, it is not even necessary to know what those interactions are. However, if one has a good microscopic model of the system then it may sometimes be useful to calculate the partial molar quantities of the system based on the properties of the model, including the interactions between the components, and much of this paper is devoted to analyzing the thermodynamic properties of microscopic models using partial molar quantities.

A second property of partial molar quantities is that even though an extensive property of a system such as entropy or volume may be constrained by physical considerations to be positive, one or more of the partial molar quantities of a system may be positive, zero, or even negative, as long as the sum of the terms, e.g. in Equation (2), is positive. For example, the partial molar volume of $\mathrm{MgSO}_{4}$ in dilute aqueous solution is negative [4], and the partial molar entropy of aqueous $\mathrm{Mg}_{3}\left(\mathrm{PO}_{4}\right)_{2}$ in its standard state is negative with the rather astounding negative value of $-831.8 \mathrm{~J} \cdot \mathrm{K}^{-1} \cdot \mathrm{mol}^{-1}[5]$.

Partial molar quantities have not been widely used in the field of solid state physics. One exception is the Fermi level, which is the statistical mechanical equivalent of the partial molar Gibbs free energy.The present paper will explore the application of partial molar methods to electrons in metals and superconductors, in particular the partial molar entropy and the partial molar heat capacity. The information one can glean from this is complementary to other approaches to studying the thermodynamics of metals and superconductors and can provide additional insight into the thermodynamic properties and electronic structures of those materials. Furthermore, the application of partial molar quantities to solid state physics has direct relevance to long-standing problems in other fields, such as the thermodynamics of ions in solution and the thermodynamics of biological energy transformations.

\section{Dense Electron Systems in the Low-Temperature Limit}

It is well known concept that in the low-temperature limit the total electronic entropy of a normal metal is

$$
S_{e, \text { total }}=K_{1} g(\varepsilon) T
$$

where the purposes of this presentation $K_{1}$ can be considered an arbitrary constant. This equation assumes that electrons can be reasonably treated as if they are in some sense non-interacting. The total low-temperature electronic heat capacity is given by

$$
C_{e, \text { total }}=T \frac{\partial S_{e, \text { total }}}{\partial T}=K_{1} g(\varepsilon) T
$$

which, for a material following Equation (3),is the same as the low-temperature total electronic heat capacity.

Using the definition presented in Equation (1), the partial molar electronic entropy is

$$
\bar{S}_{e}=\left(\frac{\partial S_{e, \text { total }}}{\partial n_{e}}\right)_{T, P}=\left(\frac{\partial\left(K_{1} g(\varepsilon) T\right)}{\partial n_{e}}\right)_{T, P}=K_{1} T\left(\frac{\partial g(\varepsilon)}{\partial n_{e}}\right)_{T, P} .
$$

In these expressions the total external pressure is held constant, as indicated in the subscripts. However, unless otherwise indicated, for simplicity let us consider that the volume is also constant, and one could therefore just as well replace $P$ with $V$ in the subscripts above, signifying constant volume. 
Making use of the chain rule and the following relations

$$
\frac{\partial n_{e}}{\partial \varepsilon}=g(\varepsilon)
$$

and

$$
g(\varepsilon)^{-1}=\left(\frac{\partial n_{e}}{\partial \varepsilon}\right)^{-1}=\frac{\partial \varepsilon}{\partial n_{e}}
$$

Equation (5) becomes

$$
\bar{S}_{e}=K_{1} T\left(\frac{\partial g(\varepsilon)}{\partial n_{e}}\right)_{T, P}=K_{1} T\left(\frac{\partial g(\varepsilon)}{\partial \varepsilon}\right)_{T, P}\left(\frac{\partial g(\varepsilon)}{\partial n_{e}}\right)_{T, P}=\frac{K_{1} T}{g(\varepsilon)}\left(\frac{\partial g(\varepsilon)}{\partial \varepsilon}\right)_{T, P}=K_{1} T\left(\frac{\partial \ln (g(\varepsilon))}{\partial \varepsilon}\right)_{T, P} .
$$

From equation (2) the partial molar entropy contributes

$$
n_{e} \bar{S}_{e}=n_{e} \frac{K_{1} T}{g(\varepsilon)}\left(\frac{\partial g(\varepsilon)}{\partial \varepsilon}\right)_{T, P}
$$

to the total entropy of the system.

Making use of

$$
n_{e}=\int_{\varepsilon_{0}}^{\varepsilon} g(\varepsilon) \mathrm{d} \varepsilon
$$

Equation (9) becomes

$$
n_{e} \bar{S}_{e}=n_{e} \frac{K_{1} T}{g(\varepsilon)}\left(\frac{\partial g(\varepsilon)}{\partial \varepsilon}\right)_{T, P}=\frac{K_{1} T}{g(\varepsilon)}\left(\frac{\partial g(\varepsilon)}{\partial \varepsilon}\right)_{T, P} \int_{\varepsilon_{0}}^{\varepsilon} g(\varepsilon) \mathrm{d} \varepsilon .
$$

Comparing the total electronic entropy (Equation (3)) to the partial molar entropy's contribution to the total (Equation (11)), one concludes that the total electronic entropy provides information on the density of states in the vicinity of the Fermi level, whereas the partial molar electronic entropy, when used in conjunction with information from the total electronic entropy, also provides information on the slope of the density of states curve in the vicinity of the Fermi level. Thus, if the partial molar entropy can be measured or otherwise determined it can provide additional insight into the electronic structure, the form of the density of states function in the vicinity of the Fermi level, and the thermodynamics of metals.

\section{Sommerfeld Model}

The Sommerfeld model is perhaps the simplest conceptual model that captures many of the essential thermodynamic features of electrons in metals. In the Sommerfeld model non-interacting Fermions are confined to rigid box of constant volume. Let us compare the total electronic entropy to the partial molar contribution to the entropy in this model. Given that the density of states in the Sommerfeld model is given by

$$
g(\varepsilon)=K_{2} \varepsilon^{1 / 2}
$$

where $K_{2}$ is a constant that for our purposes can be considered to be arbitrary, the total electronic entropy becomes

$$
S_{e, S, \text { total }}=K_{1} g(\varepsilon) T=K_{1} K_{2} \varepsilon^{1 / 2} T=K_{3} \varepsilon^{1 / 2} T
$$

where the subscript $S$ specifies "Sommerfeld model”. The contribution of the partial molar electronic heat capacity to the total heat capacity becomes

$$
n_{e} \bar{S}_{e, S}=n_{e} \frac{K_{1} T}{K_{2} \varepsilon^{1 / 2}}\left(\frac{\partial\left(K_{2} \varepsilon^{1 / 2}\right)}{\partial \varepsilon}\right)_{T, P}=\frac{K_{1} T}{K_{2} \varepsilon^{1 / 2}}\left(\frac{\partial\left(K_{2} \varepsilon^{1 / 2}\right)}{\partial \varepsilon}\right)_{T, P} \int_{\varepsilon_{0}}^{\varepsilon}\left(K_{2} \varepsilon^{1 / 2}\right) \mathrm{d} \varepsilon .
$$

Performing the derivatives and integrals this equation becomes 


$$
n_{e} \bar{S}_{e, S}=\frac{1}{3} K_{3} \varepsilon^{1 / 2} T=\frac{1}{3} S_{e, S, \text { total }} .
$$

From this we see that the partial molar electronic entropy's contribution to the total electronic entropy does not necessarily equal the total electronic entropy. In fact, in the Sommerfeld model the partial molar entropy contributes only one third of the total.

One can conclude the same thing by considering the temperature coefficient of the Fermi level. The Fermi level $\left(\varepsilon_{f}\right)$ is the statistical mechanical equivalent of the partial molar Gibbs free energy of a Fermi gas. Let $\varepsilon_{f, s}$ represent the Fermi level in the Sommerfeld model. For a degenerate free electron Fermi gas it is given by the following expression [6]:

$$
\varepsilon_{f, S}=\varepsilon_{f 0, S}\left[1-\frac{\pi^{2}}{12}\left(\frac{k T}{\varepsilon_{f 0, S}}\right)^{2}+\cdots\right]
$$

where $\varepsilon_{f 0, S}$ is the zero-temperature Fermi level and $k$ is Boltzmann's constant. From elementary chemical thermodynamics the partial molar entropy can be calculated by an indirect method, i.e. by the negative of the temperature coefficient of the partial molar Gibbs free energy:

$$
\bar{S}_{e, S}=-\frac{\partial \varepsilon_{f, S}}{\partial T}=\frac{(\pi k)^{2}}{6 \varepsilon_{f 0, S}} T .
$$

The total entropy is given by

$$
S_{e, S}=\frac{(\pi k)^{2}}{2 \varepsilon_{f 0, S}} n_{e} T .
$$

The partial molar entropy is given by the following the following direct calculation

$$
\bar{S}_{e, S}=\frac{\partial S_{e, S}}{\partial n_{e}}=\frac{(\pi k)^{2}}{6 \varepsilon_{f 0, S}} T
$$

and the partial molar contribution to the total entropy is given by

$$
n_{e} \bar{S}_{e, S}=n_{e} \frac{\partial S_{e, S}}{\partial n_{e}}=\frac{(\pi k)^{2}}{6 \varepsilon_{f 0, S}} n_{e} T .
$$

From these equations one can see by inspection that

$$
n_{e} \bar{S}_{e, S}=\frac{S_{e, S}}{3}
$$

which is the same result as Equation (15).

Where is the "missing" two thirds of electronic entropy? Considering that the Sommerfeld model contains only two components, electrons and lattice, the missing two thirds must be assigned to the lattice! More specifically, in the Sommerfeld model partial molar lattice entropy contributes two thirds of the total electronic entropy, and the partial molar electronic entropy contributes one third of the total electronic entropy.

This result may seem counterintuitive. In the Sommerfeld model there are no modes of motion of the lattice which can absorb thermal energy, so naively one might expect that it cannot contribute to the entropy. However, one must keep in mind what is meant by "non-interacting" in the Sommerfeld model. Although it is true that electrons do not interact with each other, they do interact strongly with the lattice because the lattice confines the electrons. This interaction causes the partial molar entropy of the lattice to contribute to the total electronic entropy in the sense defined by Equation (2).

In the Sommerfeld model the "lattice" can be thought of as empty space terminated by walls. The partial molar lattice entropy is the change in entropy upon the addition of a volume element. If the volume of the system is incremented by a volume element the electron gas becomes more dilute, hence the entropy increases, and this accounts for the fact that the partial molar lattice entropy contributes two thirds of the total entropy in the Sommerfeld model, even though the lattice has no modes of motion itself. 
Dividing the entropy in this manner into contributions from the components of the system might seem to be just a mathematical formality, but it is much more than this. Because the partial molar entropy determines how the entropy of a system changes when one component is added to the system it tells us how the entropy of a metal changes when adding an electron as well as how the entropy changes when adding a lattice component. This in turn tells us the entropy of a process wherein an electron is transferred between two metals.

\section{Band Theory}

Things become more interesting when we consider the electronic entropy from the point of view of a band theory, which begins to better approximate the properties of real metals than does the Sommerfeld model. It will be shown that in band theory not only can partial molar electronic entropy be different from the total electronic entropy, but it can even be negative.

Assume the following: Conduction band electrons interact with a fixed periodic pseudopotential representing the lattice, i.e. nuclei and core electrons. There is no direct electron-electron interaction. The density of states function, $g(\varepsilon)$, is independent of the number of electrons in a band, i.e. neither the filled nor empty energy levels are perturbed by the addition of a small number of additional electrons. The electron density is high enough and the temperature is low enough that the thermodynamics of the system behaves as a strongly-degenerate nearly free electron gas. Also, the Debye heat capacity will be ignored in this section.

In general, from a statistical mechanical perspective the conduction band of a metal can be characterized in terms of a density of states function, separated from above and below by band gaps. The density of states function is zero at the low-energy edge of the band, climbs to at least one maximum, and then decreases to zero at the high-energy edge or the band. This implies that there is at least one region of positive slope, one region of negative slope, and one point of zero slope on the $\varepsilon$ vs. $g(\varepsilon)$ curve. These simple facts have profound implications for the nature of partial molar electronic entropies.

Consider the mathematical properties implied by the facts just cited as applied to Equation (8), repeated here as Equation (22):

$$
\bar{S}_{e, B}=\frac{K_{1} T}{g(\varepsilon)}\left(\frac{\partial g(\varepsilon)}{\partial \varepsilon}\right)_{T, P} .
$$

The right hand side can be broken into three factors

$$
\bar{S}_{e, B}=F_{1} F_{2} T
$$

where $F_{1}$ depends on the density of states,

$$
F_{1}=\frac{K_{1}}{g(\varepsilon)}
$$

and $F_{2}$ is the slope of the density of states curve

$$
F_{2}=\left(\frac{\partial g(\varepsilon)}{\partial \varepsilon}\right)_{T, P} .
$$

The density of states is physically constrained to be non-negative at every point within a band, implying that $g(\varepsilon)$ is everywhere non-negative and $F_{1}$ must therefore be non-negative at every point on the $g(\varepsilon)$ curve. However, as noted above the slope of the curve may be positive, negative, or zero, implying that $F_{2}$ may be positive negative, or zero. This in turn implies that the partial molar electronic entropy (Equation (23)) may be positive, negative or zero.

This is illustrated in Figure 1 through Figure 3 which show the density of states curves for the conduction band for three idealized cases. For the purposes of this discussion, which is to illustrate the general features of partial molar electronic entropies in comparison to total electronic entropies, it is sufficient that the units in these figures to be arbitrary units. It is also not necessary that these conceptual models of bands be completely realistic, but only that the slopes in the density of states curve has the feature of having one or more regions each of positive, negative, and zero slope. The area under each curve (which gives the maximum possible electrons in the band) is the same for each curve, and in Figure 1 and Figure 2 the band is half full, as indicated by the darkened 
regions. The $y$-axis of each curve represents the density of states, and the x-axis represents energy. At the Fermi level the density of states is the same for Figure 1 and Figure 2. The shapes of the density of states curves in Figure 1 and Figure 3 are the same, and the shape of the curve in Figure 2 is the mirror image of the shape of the curves in Figure 1 and Figure 3.

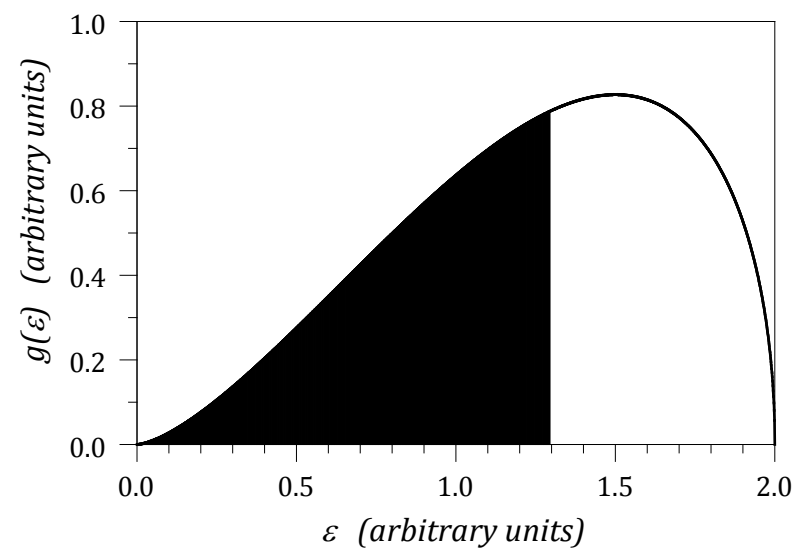

Figure 1. Density of states curve for hypothetical band \#1, half filled. Slope is positive at the Fermi level.

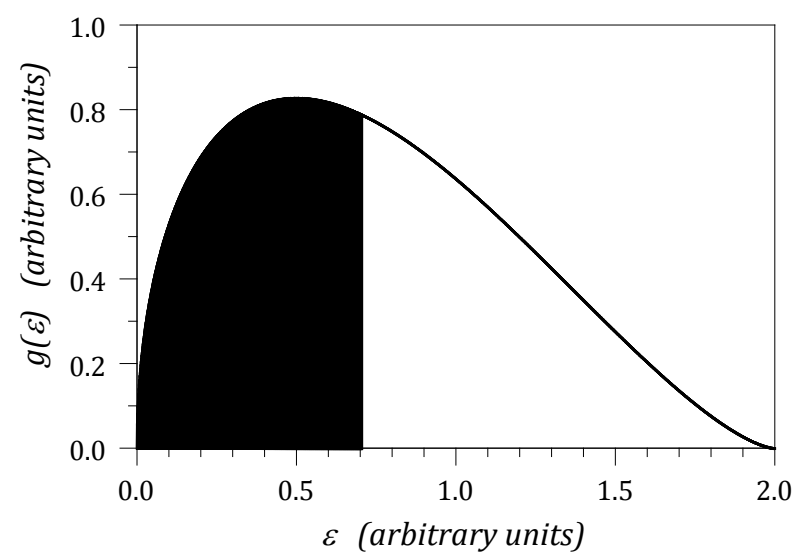

Figure 2. Density of states curve for hypothetical band \#2, half filled. Slope is negative at the Fermi level.

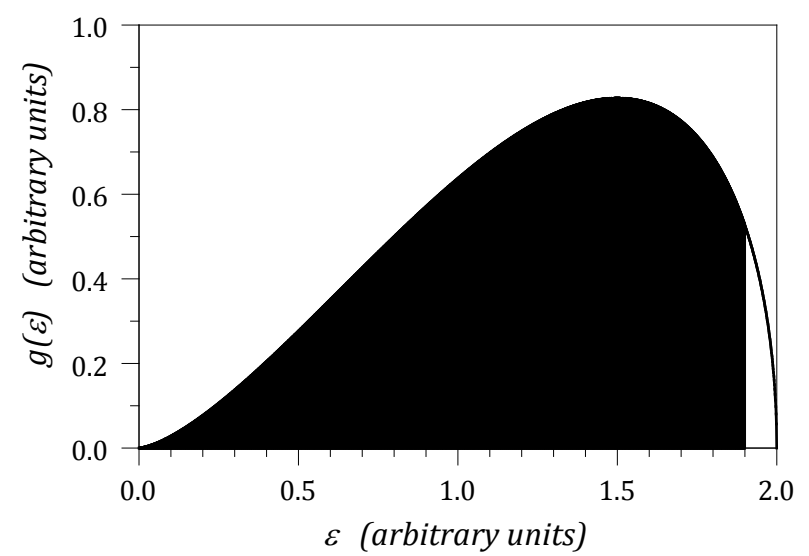

Figure 3. Density of states curve for a hypothetical band \#1, nearly filled. Slope is strongly negative at the Fermi level. 
The total electronic heat capacity for Figure 1 and Figure 2 is

$$
S_{e, \text { total }}=K_{1} g(\varepsilon) T=0.7887 K_{1} T .
$$

The contribution of the partial molar electronic entropy to the total electronic entropy in Figure 1 is

$$
n_{e} \bar{S}_{e}=\frac{K_{1} T}{g(\varepsilon)}\left(\frac{\partial g(\varepsilon)}{\partial \varepsilon}\right)_{T, P} \int_{\varepsilon_{0}}^{\varepsilon} g(\varepsilon) \mathrm{d} \varepsilon=0.2245 T
$$

The partial molar electronic heat capacity contributes only $28.46 \%$ of the total electronic heat capacity.

The contribution of the partial molar electronic heat capacity to the total electronic heat capacity in Figure 2 is

$$
n_{e} \bar{S}_{e}=-0.2245 T \text {. }
$$

The partial molar contribution to the total is $-28.46 \%$ in this case, i.e. a negative contribution.

Figure 3 represents an extreme case. The density of states curve is the same as for Figure 1, but in this case the band is nearly full. The slope of the density of states curve is a large negative value, implying a comparatively large negative value for the partial molar electronic heat capacity. This example would also be expected to show a negative effective electron mass [7]. It is also possible for the partial molar electronic entropy to be zero. This would happen if the slope of the density of states curve were zero at the Fermi level.

Figure 4 shows a somewhat more realistic band shape [8]. Noted on the figure are three possible locations for the Fermi level. The three levels are not far apart on the density of states curve, but the partial molar electronic entropies will be very different for each of the three cases. For $\varepsilon_{f 1}$, the partial molar entropy will be positive and comparatively large because the slope is positive and the magnitude of the slope is comparatively large. For $\varepsilon_{f 3}$ the partial molar entropy will be negative with a comparatively large magnitude. For $\varepsilon_{f 2}$, the Fermi level in on the cusp of the curve. The total electronic entropy would be a maximum at that point because the density of states curve is at a maximum, but the partial molar entropy would be very sensitive to the exact placement of the Fermi level and therefore difficult to predict. This is because it is at the cusp of the curve where the slope abruptly changes from a comparatively large positive value to a comparatively large negative value. However, if $\varepsilon_{f 2}$ is perfectly centered on the cusp (at a point of zero slope) then the partial molar electronic entropy will be zero. Density of states curves for real metals can be much more complicated than those in the illustrations in this paper, in some cases with multiple maxima and minima [9], and the partial molar entropies will depend on exactly where the Fermi level would fall on these complicated structures.

Thus, we see that both positive and negative values for the partial molar electronic entropy are consistent with band theory, and these values depend on the properties of the density of states curve in the vicinity of the zerotemperature Fermi level. Consequently, partial molar electronic heat capacities can tell us a significant amount of information about the shape of the conduction band of a metal in the vicinity of the Fermi level.

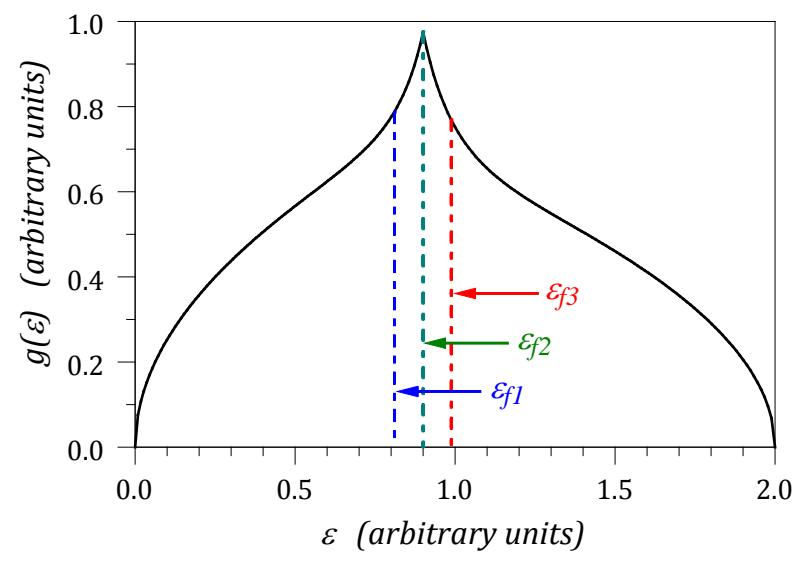

Figure 4. A conduction band of a somewhat more realistic shape than the bands in Figures 1-3. Three possible positions of the Fermi level are shown. 


\section{Debye Heat Capacity}

We conventionally think of the low temperature electronic heat capacity and entropy as being simply proportional to $T$, e.g. Equation (3). However, Equation (3) is only the lowest term in a power series expansion of the total electronic entropy, and higher order terms, including one proportional to $T^{3}$ also exist. This would apply to both the total electronic heat capacity and the partial molar electronic heat capacity, though not necessarily equally. Thus, although we often ascribe any $T^{3}$ term in the total heat capacity to Debye heat capacity, part of the $T^{3}$ term comes from the series expansion of the Fermi-Dirac distribution and is therefore at least partly electronic in nature. However, given the assumption that the electron system in a metal is strongly degenerate, it is reasonable to assume that these higher order terms in this series expansion are negligible compared to the term linear in $T$ and can be ignored.

However, there is another effect that can also lead to a $T^{3}$ term in the partial molar electronic heat capacity, and this arises from the role of electrons in the Debye theory. The Debye heat capacity, $C_{D}$, at low temperature is given by [10]

$$
C_{D}=\frac{12 N k \pi^{4}}{5}\left(\frac{T}{T_{D}}\right)^{3}+\cdots
$$

where $N$ is the number of lattice elements and $T_{D}$ is the Debye temperature characteristic of the metal. The low-temperature the Debye entropy is, by integration

$$
S_{D}=\int_{0}^{T} T^{-1} C_{D} \mathrm{~d} T=\frac{C_{D}}{3}=\frac{4 N k \pi^{4}}{5}\left(\frac{T}{T_{D}}\right)^{3}+\cdots=\frac{C_{D}}{3}+\cdots .
$$

The partial molar electronic Debye heat capacity at low temperature is

$$
\bar{C}_{e, D}=\frac{\partial C_{D}}{\partial n_{e}}=\left(\frac{\partial C_{D}}{\partial T_{D}}\right)\left(\frac{\partial T_{D}}{\partial n_{e}}\right)=\frac{-36 N k \pi^{4}}{5 T_{D}}\left(\frac{\partial T_{D}}{\partial n_{e}}\right)\left(\frac{T}{T_{D}}\right)^{3}+\cdots=-3\left(\frac{C_{D}}{T_{D}}\right)\left(\frac{\partial T_{D}}{\partial n_{e}}\right)+\cdots
$$

The partial molar electronic Debye entropy at low temperature is, by integration,

$$
\bar{S}_{e, D}=\int_{0}^{T} \frac{\bar{C}_{e, D}}{T} \mathrm{~d} T=\frac{-12 N k \pi^{4}}{5 T_{D}}\left(\frac{\partial T_{D}}{\partial n_{e}}\right)\left(\frac{T}{T_{D}}\right)^{3}+\cdots=-3\left(\frac{S_{D}}{T_{D}}\right)\left(\frac{\partial T_{D}}{\partial n_{e}}\right)+\cdots .
$$

In the rest of this paper, the higher order terms will be dropped from the low-temperature limiting expressions unless otherwise indicated. From this equation one sees that (unless $\partial T_{D} / \partial n_{e}=0$ ) the partial molar electronic entropy contains a term proportional to $T^{3}$ arising from the dependence of Debye heat capacity on the number of electrons.

Shifting attention to the high-temperature limit, the Debye heat capacity is given by

$$
C_{D}=3 N k\left(1-\frac{1}{20}\left(\frac{T_{D}}{T}\right)^{2}+\cdots\right) \text {. }
$$

The partial molar heat capacity in the high-temperature limit is therefore

$$
\frac{\partial C_{D}}{\partial n_{e}}=\frac{-3 N k}{10 T_{D}}\left(\frac{T}{T_{D}}\right)^{-2} \frac{\partial T_{D}}{\partial n_{e}}+\cdots
$$

The higher order terms will be dropped from this expression in the rest of the paper unless otherwise indicated

From this equation, one can see that at high temperature the Debye heat capacity contributes to the partial molar electronic heat capacity through a term proportional to $T^{-2}$, which asymptotically approaches zero in the limit of high temperature.

The word "electronic" in the phrases "partial molar electronic Debye heat capacity" and "partial molar electronic Debye entropy" is not meant to conceptually substitute electronic motion for vibrational motion in the Debye theory, but rather to recognize that the Debye heat capacity must vary as electrons are added to or removed from the metal. 
That the Debye heat capacity could contribute to the partial molar electronic heat capacity may seem counter-intuitive at first. However, on must keep in mind that the density and elastic constants of a metal depend on the electronic structure of the metal. When an electron is added to a metal it enters the conduction band. Depending on the electronic structure of the metal the added electron may go into an energy level that is, using a chemists' conceptual picture, either bonding or anti-bonding in character. (As a special case it may also be neutral with respect to bonding properties.) This could affect the crystal in two ways: changing the average interatomic distance (hence the density) and/or changing the elastic constants of the material. These parameters determine phonon spectrum of the material, which in turn determines the Debye temperature. Adding or removing electrons from the material will therefore alter these parameters, hence changing the heat capacity and entropy. One must conclude that in general the Debye heat capacity contributes to the partial molar entropy of the material. This condition would only be violated in cases where there is an accidental cancellation of terms.

The effect of electronic structure on the phonon spectrum can be considered a form of electron-phonon interaction. Usually electron-phonon interactions are treated in terms of scattering theory, but as we see here one can also learn something about electron-phonon interactions from the equilibrium thermodynamic properties of the system, specifically the partial molar electronic Debye entropy. One limitation of this model as applied to real metals is that the Debye temperature is not a temperature-independent quantity, so the treatment given here should be considered only a first approximation.

What magnitude might one expect from this effect? Without additional information from experimental or theoretical studies it is hard to predict. However, Let us break down the factors in the partial molar electronic Debye heat capacity and entropy to see what can be learned from their functional forms. In the low-temperature limit (Equations (31) and (32)) we have

$$
\begin{aligned}
& \bar{C}_{e, D}=-3\left(\frac{C_{D}}{T_{D}}\right)\left(\frac{\partial T_{D}}{\partial n_{e}}\right) \\
& \bar{S}_{e, D}=-3\left(\frac{S_{D}}{T_{D}}\right)\left(\frac{\partial T_{D}}{\partial n_{e}}\right) .
\end{aligned}
$$

These are just rescaled versions of the Debye heat capacity and the Debye entropy. Both are rescaled by $T_{D}^{-1}$ and $\partial T_{D} / \partial n_{e}$. One of these scale factors, $T_{D}^{-1}$, is straightforward. It is simply

$$
T_{D}=\frac{h v_{m}}{k}
$$

where $h$ is Planck's constant, $k$ is Boltzmann's constant, and $v_{m}$ is the Debye cutoff frequency.

To calculate $\partial T_{D} / \partial n_{e}$ we start with the definition of $T_{D}$, and the factor $\partial T_{D} / \partial n_{e}$ becomes

$$
\frac{\partial T_{D}}{\partial n_{e}}=\frac{h}{k}\left(\frac{\partial v_{m}}{\partial n_{e}}\right) .
$$

The cutoff frequency in Debye theory is defined by

$$
v_{m}=\left(\frac{9 N}{4 \pi V\left(a_{L}^{-3}+2 a_{T}^{-3}\right)}\right)^{1 / 3} .
$$

Therefore using these relations and differentiating using the chain rule we have

$$
\frac{\partial T_{D}}{\partial n_{e}}=\frac{h}{k}\left(\frac{\partial v_{m}}{\partial n_{e}}\right)=\left(\frac{4 \pi k^{3} V}{9 N h^{3}}\right) T_{D}^{4}\left(a_{L}^{-4} \frac{\partial a_{L}}{\partial n_{e}}+2 a_{T}^{-4} \frac{\partial a_{T}}{\partial n_{e}}\right)
$$

where $N$ is the number of lattice sites in the crystal, $V$ is the volume of the crystal, $a_{L}$ is the longitudinal speed of sound, and $a_{T}$ is the transverse speed of sound. Therefore

$$
\bar{C}_{e, D}=-C_{D}\left(\frac{4 \pi k^{3} V}{3 N h^{3}}\right) T_{D}^{3}\left(a_{L}^{-4} \frac{\partial a_{L}}{\partial n_{e}}+2 a_{T}^{-4} \frac{\partial a_{T}}{\partial n_{e}}\right)
$$


and

$$
\bar{S}_{e, D}=-S_{D}\left(\frac{4 \pi k^{3} V}{3 N h^{3}}\right) T_{D}^{3}\left(a_{L}^{-4} \frac{\partial a_{L}}{\partial n_{e}}+2 a_{T}^{-4} \frac{\partial a_{T}}{\partial n_{e}}\right) .
$$

The longitudinal speed of sound depends on the bulk modulus, the shear modulus, and the density. The transverse speed of sound depends on shear modulus and the density. Therefore, $\partial a_{L} / \partial n_{e}$ and $\partial a_{T} / \partial n_{e}$ in these equations can be replaced by

$$
\frac{\partial a_{L}}{\partial n_{e}}=\frac{\partial a_{L}}{\partial \mu_{B}} \frac{\partial \mu_{B}}{\partial n_{e}}+\frac{\partial a_{L}}{\partial \mu_{S}} \frac{\partial \mu_{S}}{\partial n_{e}}+\frac{\partial a_{L}}{\partial \rho} \frac{\partial \rho}{\partial n_{e}}
$$

and

$$
\frac{\partial a_{T}}{\partial n_{e}}=\frac{\partial a_{T}}{\partial \mu_{S}} \frac{\partial \mu_{S}}{\partial n_{e}}+\frac{\partial a_{T}}{\partial \rho} \frac{\partial \rho}{\partial n_{e}}
$$

where $\mu_{B}$ is the bulk modulus, $\mu_{S}$ is the shear modulus, and $\rho$ is the density. The longitudinal and transverse speeds of sound are given by

$$
a_{L}=\left(\frac{\mu_{B}+\frac{4 \mu_{S}}{3}}{\rho}\right)^{1 / 2}
$$

and

$$
a_{T}=\left(\frac{\mu_{S}}{\rho}\right)^{1 / 2}
$$

respectively, so the partial derivatives $\partial a_{L} / \partial \mu_{B}, \partial a_{L} / \partial \mu_{S}, \partial a_{L} / \partial a_{L}, \partial a_{T} / \partial \mu_{S}$ and $\partial a_{T} / \partial \rho$ are calculable from using macroscopic measurements. The partial derivatives $\partial \mu_{B} / \partial n_{e}, \partial \mu_{S} / \partial n_{e}, \partial \rho / \partial n_{e}, \partial \mu_{S} / \partial n_{e}$, and $\partial \rho / \partial n_{e}$ are accessible via electronic structure calculations, at least in principle. It might be expected that these will be relatively small numbers, so the partial molar electronic Debye heat capacity and entropy may be a small fraction of the total Debye heat capacity and entropy. However, even a small fraction of the Debye heat capacity may represent a fairly large contribution to the partial molar electronic heat capacity, given the fact that the electronic heat capacity is quite small for metals, especially at moderately low and low temperatures.

\section{Superconductors}

A two-fluid conceptual model is often applied to superconductors. Usually this is applied to explain the property of zero DC resistance [2] [11]. However, let us consider the thermal properties of superconductors from the point of view of a two-fluid model. Electrons in a superconductor are grouped into two categories: normal electrons and superconducting electrons. In BCS theory superconducting electrons exist as a Bose condensate of Cooper pairs resulting from electron-phonon interactions. Below the superconducting transition temperature both normal and superconducting electrons coexist in the same sample.

To use an analogy, one might compare a superconductor to a system composed of a sealed vessel containing a small amount of vapor and a condensate of the vapor. Above a certain temperature (the dew point) only the vapor exists, but below the dew point both phases coexist within the sealed vessel. Below the dew point the number density of the vapor is determined by vapor pressure of the condensate. In this analogy, the dew point corresponds to the critical temperature for the normal/superconductor transition.

Starting from a temperature above the dew point, as the temperature drops the system eventually reaches the dew point and a condensed phase begins to form, and as the temperature drops still further a greater fraction of the material will leave the vapor and enter the condensed phase. At $T=0$ all of the material exists as a condensate. In a superconductor this behavior would be analogous to a greater fraction of conduction electrons existing in the Bose condensate as temperature is lowered.

If one were unaware of the fact that two phases may exist inside of the sealed container, and if one were to 
perform a heat capacity measurement on the system one would observe a discontinuity at the dew point. Above the dew point the heat capacity is that of the vapor alone. Just below the dew point the apparent heat capacity is dominated by the latent heat for the phase transition between vapor and condensate, i.e. as one cools the temperature there is a condensation of vapor, and the latent heat is given up, which implies that to reduce the temperature an "unexpectedly" large amount of heat removal would be required. Thus, immediately below the transition temperature the apparent heat capacity is greater than that of the vapor. This qualitatively resembles the temperature vs. heat capacity curve for a superconductor.

As the temperature approaches zero the number density of the vapor drops in exponential-like fashion. This is because the concentration of the vapor pressure drops in exponential-like fashion; hence there are fewer vapor molecules to condense as the temperature drops. This qualitatively resembles the drop in number density of normal electrons in a superconductor as temperature is reduced toward zero.

At very low temperature there is not much vapor left, so the heat capacity of the vapor is negligible. Furthermore, because there is little vapor left to condense latent heat available by condensation is also negligible. At that point the apparent heat capacity of the system is largely dominated by the heat capacity of the condensed phase. For a superconductor the condensate is highly ordered Bose condensate, which has a heat capacity of zero. As the temperatures approaches zero the heat capacity of a superconductor approaches zero with a zero slope.

There is nothing very startling in this analogy so far, but what if we were to extend the analogy? In the dew-in-can analogy, below the dew point the number density of the gas is fixed by the vapor pressure of the gas, and adding an additional quantity of condensate will not change the number density of the gas but rather all added molecules enter the condensed phase. Furthermore, if we assume that the volume of condensate is small compared to the volume of the vessel then the total number of vapor molecules will be essentially unchanged if a small amount of condensate is added.

What if a superconductor were to behave the same way? In other words, at a given temperature the normal electrons would exist in equilibrium with the Bose condensate. The idea of an equilibrium between a gas-like state (normal electrons) and a liquid-like state (Bose condensate) was discussed briefly by Kittel [2]. Pursuing this line of thought further, let us assume that the number density of normal electrons would be fixed by a something analogous to a vapor pressure, regardless of how many electrons would be in the Bose condensate. In this case, if electrons were to cross the phase boundary and enter the superconductor they would all go into the Bose condensate, leaving the number of normal electrons unaltered. Since the Bose condensate is a state of zero entropy, adding electrons would not change the entropy of the system. This would imply that the partial molar electronic entropy of a superconductor would be zero, and by implication the partial molar heat capacity would also be zero.

Thus, in this conceptual model for a superconductor there would be a non-zero total electronic heat capacity and a non-zero total electronic entropy, but the partial molar electronic heat capacity and partial molar electronic entropy would both be zero. This also relates to the Fermi level. The temperature coefficient of the partial molar Gibbs free energy (the Fermi level for electrons) is equal to the negative of the partial molar entropy. Therefore, if, as suggested here, the partial molar electronic entropy of a superconductor is zero it would also imply that the Fermi level of a superconductor is independent of temperature. It would be interesting to see if this prediction can be reproduced in BCS theory.

In the discussion of superconductors it is important to re-emphasize the underlying concept that superconductivity has its origin in electron-lattice interactions, in this case via electron-phonon interactions. Therefore, one needs to keep in mind that the partial molar electronic heat capacity and entropy will depend on those interactions, and "unexpected" results may occur. In this case the "unexpected" result is that the electronic entropy is zero, even at non-zero temperature, despite the existence of normal electrons in the material at finite temperature, provided that one defines "electronic entropy" as "partial molar electronic entropy".

\section{Determining Partial Molar Electronic Entropies and Heat Capacities}

As has been mentioned already, partial molar electronic heat entropies and heat capacities are accessible through electronic structure calculations, at least in principle. This assumes that one would calculate not only the density of electronic states at the equilibrium interatomic distances, but also the elastic constants of the material. However, accurate calculations are likely to be difficult, and in any case science is ultimately an empirical enterprise, 
which brings up the question of how one might experimentally measure partial molar electronic entropies and heat capacities.

Appealing directly to the definition of the partial molar quantities, one way to measure the partial molar electronic heat capacity of a metal would be to perform two heat capacity measurements on the same sample, one before and one after the addition or removal of a small number of electrons. The partial molar heat capacity would be the difference divided by the number of electrons added or removed. The partial molar entropy could in turn be calculated by integration. However, as a practical matter one cannot add or remove enough electrons to make a noticeable difference in the heat capacity. Therefore, one must search for alternative approaches for experimental measurements.

One alternative approach is through the measurement of thermoelectric coefficients [12]-[15]. Using the laws of reversible and irreversible thermodynamics one can show that the absolute thermoelectric power and the partial molar electronic entropy are related by the following equation:

$$
\bar{S}_{e}=-\mathcal{F} \sigma+A(T)
$$

where $\mathcal{F}$ is Faraday's constant, $\sigma$ is the absolute Seebeck coefficient, also known as the absolute thermoelectric power, and $A(T)$ is a universal function of temperature, i.e. the same for all materials [12]-[15]. Because Equation (47) can be derived from macroscopic thermodynamics its validity is independent of any microscopic models. Also because it is derived from macroscopic thermodynamics and contains only thermodynamic functions it is a thermodynamic relationship. This of course assumes that $A(T)$ can be classed as a thermodynamic function, but since it arises from a thermodynamic analysis it seems reasonable to characterize it as a thermodynamic function, even if we do not know what its value is. We do know however that it must have the same dimensionality as entropy.

It is impossible to evaluate $A(T)$ using the known laws of thermodynamics, whether equilibrium or nonequilibrium and whether used alone or in combination. However, considering that $A(T)$ is a universal function it is only necessary to determine it for a single material, so one might as well take the easiest approach possible. Therefore, let us step outside of the realm of macroscopic thermodynamics and evaluate $A(T)$ using statistical mechanics applied to one or more specific and simple microscopic models. Using this approach one can conclude that

$$
A(T)=0 .
$$

The reason that Equation (48) is valid is that both the electronic entropy and the thermoelectric power both approach zero as the density of a degenerate free electron gas approaches infinity [13].

From Equations (47) and (48) we conclude that,

$$
\bar{S}_{e}=-\mathcal{F} \sigma
$$

or in other words, the absolute thermoelectric power is essentially equivalent to the partial molar electronic entropy of a conductor. Tykodi has reached the same conclusion using other methods [15]. Since partial molar capacity is related to partial molar entropy by the expression

$$
\bar{C}=T \frac{\partial \bar{S}}{\partial T}
$$

and the Thomson coefficient, $\tau$, is related to absolute thermoelectric power by the same equation

$$
\tau=T \frac{\partial \sigma}{\partial T} \text {. }
$$

by differentiating Equations (49) and substituting Equations (50) and (51) into the result we have

$$
\bar{C}_{e}=-\mathcal{F} \tau
$$

or in other words, the Thomson coefficient is essentially equivalent to the partial molar electronic heat capacity of a conductor.

Equivalence between thermoelectric coefficients and equilibrium thermodynamic quantities was first proposed by William Thomson more than a century and a half ago, who suggested that the Thomson coefficient might be considered the specific heat of electricity. In more modern form, when framed in terms of partial molar 
quantities, Thomson's conjecture is given by Equation (52).

Thomson's conjecture, along with Equation (49) to which it is closely related, has been extremely controversial ever since he proposed it. There are two basic objections to this idea. One is that it is impossible to prove using the laws of thermodynamics. The other is that it would imply the existence of negative electronic entropies and heat capacities, since the thermoelectric coefficients may be of either sign [16].

Considering the second objection, it fails to take into account that a proper statement of the relationship between thermoelectric coefficients and their analogous equilibrium thermodynamic functions must be framed in terms of partial molar quantities, and partial molar quantities are not constrained to be non-negative quantities. In fact, it is known that partial molar entropies can be negative. This was pointed out earlier in this paper, i.e. the partial molar entropy of aqueous $\mathrm{Mg}_{3}\left(\mathrm{PO}_{4}\right)_{2}$ in its standard state is extremely negative. Furthermore, as also pointed out earlier in this paper, negative partial molar entropies are consistent with the band theory of solids.

Considering the first objection, it is logically unsound because a failure to prove something is not equivalent to disproving it. While it is true that Equations (49) and (52) have never been proven using the laws of reversible and irreversible thermodynamics, and one can even concede that it is very likely impossible to prove the relationships within the existing laws of thermodynamics, it is equally true that the relationships have never been disproven using the existing laws of thermodynamics, nor by any other method for that matter. Therefore, the matter cannot be settled under a "failure to prove" objection.

In this respect the situation can be thought of as analogous the state of affairs in the early days of thermodynamics. Before the acceptance of the second law it would have been impossible to prove or disprove it using the then-existing laws of thermodynamics. In fact, if it were possible to do so then the second law would be at theorem rather than an independent law. The same applies to the third law of thermodynamics.

With respect to the problem at hand, one way to settle the matter is to step outside of the laws of thermodynamics and apply microscopic theory to the problem. Using this approach it is possible to validate the relationships given in Equations (49) and (52) [13] [14]. As already discussed, it is only necessary to do this for just one material to validate it for all cases.

Equations (49) and (52) are relationships between macroscopic thermodynamic quantities. They connect the two theories of equilibrium and non-equilibrium thermodynamics by equating certain quantities in these two theories, therefore uniting the two in ways not possible before. Furthermore, since the relationships cannot be proven within existing thermodynamic theory, if they are valid then their existence necessarily implies the existence of a previously unrecognized law or principle of thermodynamic theory. Otherwise it would be possible to prove or disprove the relationships using the existing laws of thermodynamics.

Another alternative approach to measuring $\bar{S}_{e}$ might be to use an electron gun and an electron monochrometer. The scheme would be to bombard a sample of a metal with a low energy electron beam of very low current. For practical reasons this would be carried out in vacuum. The energy of the electron beam would be selected at high resolution, and the kinetic energy would be scanned to simulate a thermal distribution of energies. The heat released in this process would be measured at each kinetic energy of the electron beam using a calorimeter. Because the electron beam is energy resolved and designed to simulate thermal energies it might, in principle, be possible to deconvolute the partial molar electronic entropy from the data by calculation to simulating an equilibrium process for transfer of electrons between a hypothetical electron gas of known properties and the metal. However, it may be difficult for this scheme to be carried out with enough precision and accuracy to be practical.

\section{Applications and Discussion}

Partial molar electronic entropies can be used to determine the electric field inside of a conductor placed in a thermal gradient [14]. Domenicali has shown that there is a relationship between the temperature coefficient of the electrochemical potential and the electric field existing within a conductor placed in a temperature gradient [17]. The key equation is

$$
\frac{\partial \phi}{\partial x}=\frac{1}{\mathcal{F}}\left(\frac{\partial \varepsilon_{f}}{\partial T}+S_{e}^{*}\right) \frac{\partial T}{\partial X}
$$

where $\phi$ is the electrostatic potential, and $S_{e}^{*}$ is the "transported entropy" defined by

$$
S_{e}^{*}=-\mathcal{F} \sigma \text {. }
$$


However, since $\varepsilon_{f}$ is the partial molar Gibbs free energy of the electron, the partial molar entropy of the electron is

$$
S_{e}=-\frac{\partial \varepsilon_{f}}{\partial T}
$$

Substituting (54) and (55) into (53) we get

$$
\frac{\partial \phi}{\partial x}=\frac{-1}{\mathcal{F}}\left(S_{e}+\mathcal{F} \sigma\right) \frac{\partial T}{\partial x}
$$

Using Equation (47) this becomes

$$
\frac{\partial \phi}{\partial x}=\frac{-A(T)}{\mathcal{F}}\left(\frac{\partial T}{\partial x}\right)
$$

Since $A(T)$ is a universal function this equation tells us that all conductors form the same electric field in their interior if subjected to a temperature gradient. Furthermore, if as argued earlier

$$
A(T)=0
$$

then

$$
\frac{\partial \phi}{\partial x}=0
$$

and the electrostatic field inside of a conductor is zero in a conductor in a temperature gradient.

Partial molar electronic entropies of metals are related to the temperature coefficient of the contact potential. The contact potential between dissimilar metals equals the difference between Fermi levels of the metals. However, since the temperature coefficient of the Fermi level is the negative of the partial molar electronic entropy, the temperature coefficient of the contact potential is given by the difference in partial molar entropies. Adsorption of gases on the surfaces of the metals, which could shift the work functions of the metals, may be a confounding factor.

The partial molar entropy can be used in the determination of partial molar entropies of ions in solution. Consider a generic electrochemical reaction

$$
\mathrm{M}_{\text {metal }} \rightarrow \mathrm{M}_{\text {solution }}^{+}+\mathrm{e}_{\text {metal }}^{-} \text {. }
$$

The entropy of this reaction can be measured from the heat of electroplating under reversible conditions. This heat is also called the electrochemical Peltier heat

$$
\Delta S=\frac{Q_{\text {reversible }}}{T} .
$$

The entropy of the same reaction is also given related to the partial molar entropies

$$
\Delta S=\bar{S}_{\mathrm{M}^{+}, \text {solution }}+\bar{S}_{\mathrm{e}^{-}, \text {metal }}-\bar{S}_{\mathrm{M}, \text { metal }} \text {. }
$$

Therefore,

$$
\frac{Q_{\text {reversible }}}{T}=\bar{S}_{\mathrm{M}^{+} \text {,solution }}+\bar{S}_{\mathrm{e}^{-} \text {,metal }}-\bar{S}_{\mathrm{M}, \text { metal }} \text {. }
$$

Of these terms, $\Delta Q_{\text {reversible }} / T$ can be measured by the electrochemical Peltier heat, $\bar{S}_{\mathrm{M}, \text { metal }}$ can be determined from third law measurements, and $\bar{S}_{\mathrm{e}^{-} \text {metal }}$ is given by Equation (49) of this paper. $\bar{S}_{\mathrm{y}^{+} \text {solution }}$ can therefore be determined by solving the above equation for $\bar{S}_{\mathrm{M}^{+} \text {solution }}$ [13] [14]. This is quite significant, since $\bar{S}_{\mathrm{M}^{+} \text {solution }}$ has long been considered a thermodynamically unmeasurable quantity.

There is a subtle point to consider when these ideas are applied to the entropy of multiply charge ions. For example, consider the following reaction.

$$
\mathrm{Al}_{\text {metal }} \rightarrow \mathrm{Al}_{\text {solution }}^{3+}+3 \mathrm{e}_{\text {metal }}^{-} \text {. }
$$

In this case the equation corresponding to Equation (63) is 


$$
\frac{Q_{\text {reversible }}}{T}=\bar{S}_{\mathrm{Al}^{3+} \text {,solution }}+3 \bar{S}_{\mathrm{e}^{-}, \text {metal }}-\bar{S}_{\mathrm{Al}, \text { metal }} \text {. }
$$

The partial molar electronic entropy appears with a factor of three in this expression because aluminum is a trivalent metal.

This also brings up the issue of how to specify the components in a metal and how that specification relates to the contributions of partial molar quantities to the total thermodynamic functions. For example, one might describe aluminum in terms of a lattice composed of $\mathrm{Al}^{+}$, together with one electron per $\mathrm{Al}^{+}$lattice element. The contribution of the partial molar entropy to the total entropy of the system would be simply $N \overline{S_{e}}$, where $N$ is the number of lattice elements. There is nothing fundamentally wrong with this, but a more conceptually appealing choice might be to describe aluminum in terms of a lattice composed of $\mathrm{Al}^{3+}$ with three electrons per aluminum ion. This corresponds to our conception of three electrons per lattice element added into a conduction band of aluminum to form the metal. This also fits into the chemical concept of trivalent aluminum, e.g. in Equation (64). In this case one would describe the contribution of the partial molar electronic entropy to the total entropy as $3 N \bar{S}_{e}$. One could also describe aluminum in terms of a lattice composed of $\mathrm{Al}^{13+}$ ions together with 13 electrons filling the core bands and conduction bands in turn. In this case the contribution of the partial molar electronic entropy to the total entropy would be $13 N \bar{S}_{e}$.

All of these descriptions of aluminum are logically and thermodynamically consistent. Each picture gives a different calculation for the contribution of the partial molar electronic heat capacity to the total heat capacity of the crystal, but any discrepancy is taken into account by a difference in the partial molar entropy of the lattice ions. Thus, the partial molar entropy of lattice ions in a description based on $\mathrm{Al}^{3+}$ is different from the partial molar entropy of lattice ions based on $\mathrm{Al}^{13+}$.

For aluminum the most useful picture is probably the one based on a lattice of $\mathrm{Al}^{3+}$, both from the point of view of how many electrons aluminum has in its conduction band and from the point of view of the chemical reactions typical of aluminum which typically involve triply-charged aluminum. However, some metals can routinely exist in more than one charge state, such as iron, and for those the choice of how to count the number of electrons may be less clear-cut. For example, in a reaction analogous to Equation (64) the iron ion may be ferric iron, $\mathrm{Fe}^{3+}$, in which case the natural choice for the number of electrons would be three, and the contribution of the partial molar electronic heat capacity to the total heat capacity of the metal could be described as $3 N \bar{S}_{e}$. However, it is also possible for iron ions to exist in a ferrous state, $\mathrm{Fe}^{2+}$, in which case it may be more convenient to count the electrons in the metal as two per lattice element. Thus, from one point of view it may be most useful to define the number of electrons in terms the number of electrons involved in a particular process.

On the other hand, the definition of $\bar{S}_{e}$ is unambiguous, i.e. independent of what picture one uses to describe the lattice. $\bar{S}_{e}$ is always based on a one-electron process. In other words it is simply the change in entropy upon the addition of a differential quantity of electrons (expressed in either particle number or moles) per unit of electrons added.

Returning to a consideration of the discontinuity in the partial molar electronic entropy at the superconducting transition temperature, it is notable that the total entropy of the material is continuous at the transition temperature. These two facts, along with Equation (2) imply that there must be a compensating discontinuity in the partial molar entropy of the lattice ions at the superconducting transition temperature. In particular, if the partial molar electronic entropy drops from a positive value to zero as the material cools through the transition temperature, then the partial molar entropy of the lattice ions must have a compensating sudden increase as one drops the temperature through the transition temperature. Conversely, if the partial molar electronic entropy is negative above the transition temperature and zero below the transition temperature then the partial molar entropy of lattice ions must have a compensating negative discontinuity as the temperature crosses the critical temperature on the cooling curve.

There are a number of biochemical processes involving the unbalanced charge transfer of ions across membranes, including oxidative phosphorylation, photosynthesis, nerve signal propagation, and muscle contraction. Full understanding of the thermodynamics of these processes requires knowing partial molar entropies of ions, and these are only experimentally accessible via processes such as Equation (64) in conjunction with knowing partial molar electronic entropies of metals.

For example, without going into specific detail, oxidative phosphorylation takes place at mitochondrial membranes. The mechanism is based on electrochemical proton gradients across the membrane. Energy is converted 
from reducing equivalents to ATP-based energy via unbalanced transfer of charged species (protons) across the membrane. These processes are understood in qualitative, or even semi-quantitative terms, but to give a fully complete and accurate energy accounting one would need to know quantities such as partial molar entropies of ions, and these are only accessible via reactions analogous to Equation (64) in combination with knowledge of partial molar electronic entropies of metals. Similarly, the evaluation of the Gibbs free energy of the process must include single ion activities or equivalent information [18].

In another application, partial molar electronic entropies can give information on the shape of the density of states curve near the Fermi level of a conductor. This was discussed earlier in this paper.

Partial molar entropies can be used to help elucidate the properties of electron-phonon interactions. One form of this interaction was discussed in section on Debye heat capacity, but there are likely a number of others as well. For example, in thermoelectricity there is a phonon drag effect. If Equations (49) and (52) are correct the phonon drag effect must also contribute to the partial molar electronic entropy and partial molar electronic heat capacity [12].

The low temperature thermal properties of aluminum illustrate some of the issues discussed above. Figure 5 illustrates the low-temperature electronic heat capacity of aluminum just above and below the superconducting phase transition. This is taken from experimental data with the $T^{3}$ dependence removed [19]. The shape of a heat capacity curve typifies that of a typical metal undergoing a phase transition into the superconducting state. Also shown on the curve is the partial molar electronic heat capacity. The partial molar electronic heat capacity was calculated using Equation (52). The Thomson coefficient for this calculation was calculated from published thermo power data for aluminum [20]. That data was for the normal state, but it is well-known that the thermoelectric coefficients are zero for superconductors, so below the superconducting transition temperature Equation (52) predicts that the partial molar heat capacity is zero. This is consistent with a two-state model as discussed earlier in this paper.

The notable features of this comparison are that the partial molar electronic heat capacity above the transition temperature is nearly linear, which is qualitatively similar to the total heat capacity curve, but the curves do not coincide. Furthermore, below the transition temperature the curves are very different.

Figure 6 shows the comparison of the total electronic entropy and the partial molar electronic entropy using data taken from the same sources as the data for Figure 5. Again, the curves are qualitatively similar above the transition temperature, except that the curves do not overlap, and there is a small $T^{3}$ component in the partial molar electronic entropy that is absent (by definition) from the total electronic entropy. Below the transition temperature the curves are very different. The zero partial molar entropy is consistent with the two-fluid model discussed earlier in this paper. It would be interesting to know if the BCS model or other models of superconductivity would predict this, but that issue will not be pursued further in this paper.

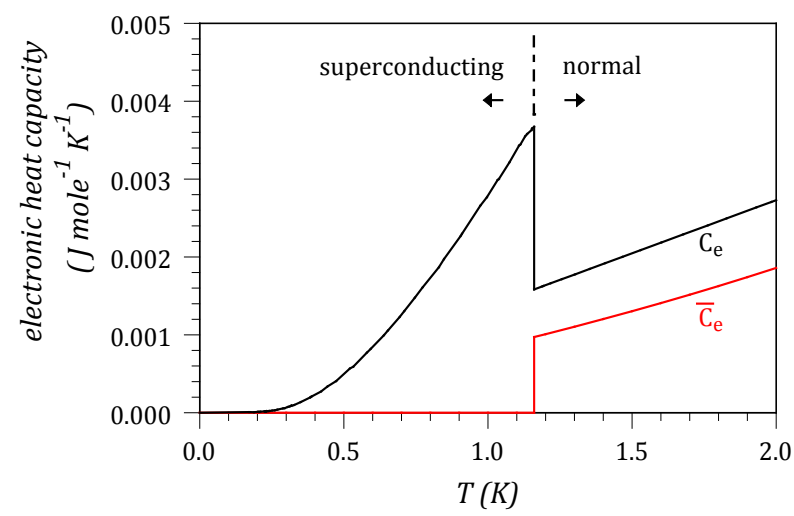

Figure 5. Partial molar electronic heat capacity $\left(\bar{C}_{e}\right)$ and total electronic heat capacity ( $C_{e}$ ) for aluminum as a function of $T$. $\bar{C}_{e}$ is based on Equation (52). $C_{e}$ is based on the conventional interpretation of electronic heat capacity used in solid state physics. Data were taken from references [19] and [20] respectively. 


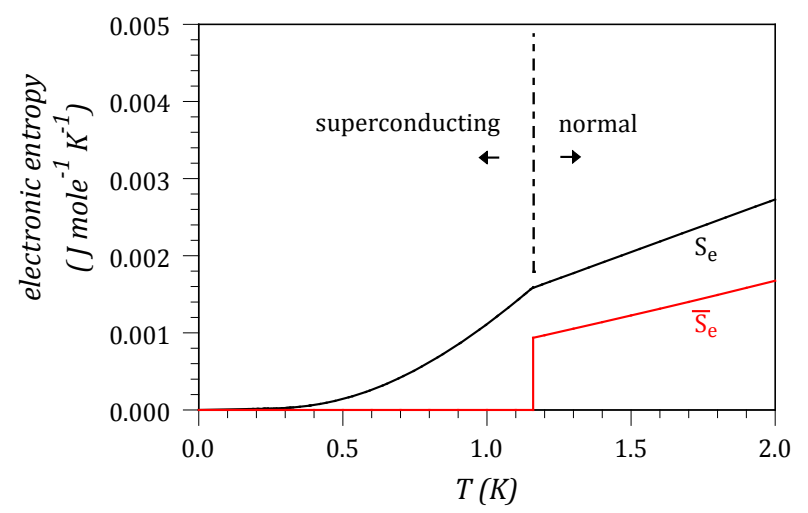

Figure 6. Partial molar electronic entropy $\left(\bar{S}_{e}\right)$ and total electronic entropy $\left(S_{e}\right)$ for aluminum as a function of $T . \bar{S}_{e}$ is based on Equation (49). $S_{e}$ is based on the conventional interpretation of electronic entropy used in solid state physics. Data were taken from references [19] and [20] respectively.

It seems clear that both the conventional approach and the partial molar approach to describing the electronic entropy and heat capacity are useful to help understand the electronic and thermal properties of metals and superconductors. It is also clear that the two approaches provide complementary information. However, there is one area in which the partial molar approach is superior. That is in dealing with the thermodynamics of process in which electrons cross phase boundaries, i.e. any process in which electrons are transferred from one material to another. The conventional way of looking at those processes cannot in general provide us with any thermodynamic information for such processes. However, partial molar quantities are inherently capable of dealing with such processes.

One conceptual hurdle is that partial molar entropies and heat capacities may be negative. Naively, this may seem to be a violation of the third law of thermodynamics, but it is not. The third law applies to total entropies, and it is known that partial molar entropies may be negative. Furthermore, it was shown that this is consistent with band theory.

As pointed out previously, one of the strengths of partial molar thermodynamics is that partial molar quantities implicitly include any interactions that might be present between the components of the system. Most commonly, thermodynamics in solid state physics are discussed in terms of non-interacting electrons or at least in terms of quasiparticles that can be thought of as free or nearly free. The quasiparticles have interactions folded into them. Generally speaking, this approach does not ask the question of what happens to the thermodynamics of the system if electrons are added or removed from the system. However, there has been some work on the interactions occurring when electrons are added or removed. For example, Hedin, Lundqvist and Lundqvist discussed the meaning of "density of states" when going beyond the one electron approximation [21]. They concentrated on a definition tied to the addition or removal of an electron. This seems to be getting close to the concepts discussed in the present paper, though they focused on spectral properties and do not seem to have applied these ideas to the calculation of partial molar quantities. One interesting point discussed in that paper is that as a result of electron-phonon interactions the density of electronic states (in the quasi-particle picture) may increase. Since this is the result of interactions between components of the system one can expect this to be reflected in the partial molar electronic entropy and heat capacity, and furthermore this effect is likely to be temperature-dependent.

This paper has pointed out some of the general features of the partial molar electronic entropy and the partial molar electronic heat capacity and applied the concepts to a few simple models. However, there are many issues not covered here. For example, not discussed are the implications of the fact that the phonon spectra of real solids do not fully conform to the assumptions of the Debye theory, in particular how these may relate to electronic structure, and the implications of thermal expansion on the electronic density of states has also not been discussed. Additionally, a more complete discussion of phonon-electron interactions has been omitted. No doubt there are many additional effects to consider and refinements that can be added to the concepts introduced here. 


\section{Summary}

The conventional way of dealing with the thermodynamics of electrons in metals and superconductors is based on the statistical mechanics of specific microscopic models. It considers the total electronic entropy and total electronic heat capacity within those models, i.e. the Sommerfeld-like heat capacity is of a normal metal is called the electronic heat capacity of the metal, and the BCS-like heat capacity of a superconductor is called the electronic heat capacity of the superconductor. These assignments provide much insight into the electronic structure and thermal properties of metals and superconductors. However, they do not fully take into account how electron-lattice interactions may affect the division of entropy and heat capacity between electrons and lattice. Instead the Sommerfeld-like heat capacity and entropy, and the BCS-like heat capacity and entropy are arbitrarily called "electronic". In addition, these approaches are entirely dependent on microscopic models. Furthermore, the conventional approach does not provide a way to analyze the thermodynamics of a process in which materials (electrons in this case) cross phase boundaries.

An alternative approach is presented here that is based on macroscopic thermodynamic principles using partial molar quantities, a rigorous approach developed by chemical thermodynamicists. This approach is model-independent in its foundations. However, it can be applied to specific microscopic models to provide additional insights about the models.

The partial molar method provides an unambiguous way to divide an extensive thermodynamic function into contributions from the components of the system as illustrated in Equation (2), and any interactions between the components of the system are automatically taken into account. Furthermore, the partial molar method provides a way to analyze the thermodynamics of a process in which a component (such as an electron) crosses a phase boundary. Thus, if an electron were to pass from a sample of iron to a sample of copper the entropy of the process would be given by

$$
\Delta S=\bar{S}_{e, \mathrm{Cu}}-\bar{S}_{e, \mathrm{Fe}}
$$

and if the process is carried out under reversible conditions the heat of the process would be given by the difference in partial molar electronic entropies.

$$
Q=T \Delta S=T\left(\bar{S}_{e, \mathrm{Cu}}-\bar{S}_{e, \mathrm{Fe}}\right) .
$$

In this paper, the partial molar method is applied to several microscopic models. It is shown that it provides additional insight into the electronic structure and thermal properties of metals and supercondcutors. In addition, a comparison is given between total electronic entropy and partial molar electronic entropy of a specific material, aluminum at low temperature. The two quantities do not coincide. A similar comparison is made between total electronic heat capacity and partial molar electronic heat capacity, and again the two quantities are quite different from each other.

The two approaches to thermodynamics of electrons are not mutually exclusive, but rather they provide complementary information. For example, the total low temperature electronic heat capacity of a normal metal tells us about the density of states at the Fermi level, whereas the partial molar electronic heat capacity and partial molar entropy provide additional information, i.e. the slope in the density of states curve at the Fermi level.

Partial molar electronic entropies have applications to a number of fields, and a few of these are specified here, including solid state physics (e.g. entropy for the process of electron transfer between materials, the properties of electrons in superconductors, and the magnitude and sign of electrostatic gradients existing in conductors in temperature gradients), chemical thermodynamics (e.g. partial molar entropies of ions in solution), and biology (e.g. energy transformation processes involving the unbalanced transfer of ions across membranes.)

In addition, the identification of the partial molar electronic entropy of a metal with the thermo power of a metal, if true, would unite the fields of reversible and irreversible thermodynamics and add an additional thermodynamic principle or law to the canon of thermodynamic theory. One possible objection to this is that it would imply the existence of negative partial molar entropies. However, negative partial molar entropies are well known in solution-phase chemistry, which shows that negative partial molar entropies do not violate fundamental thermodynamic theory, and the possible existence of negative partial molar entropies of electrons in metals is consistent with band theory.

\section{Acknowledgements and Dedication}

Partial support from ARUP Laboratories is gratefully acknowledged. I dedicate this paper to the memory of my 
parents, Linn and Elsie Rockwood.

\section{References}

[1] Kittel, C. (1976) Introduction to Solid State Physics. 5th Edition. John Wiley \& Sons, New York, 155-181

[2] Kittel, C. (1976) Introduction to Solid State Physics. 5th Edition. John Wiley \& Sons, New York, 357-398.

[3] Klotz, I.M. (1964) Chemical Thermodynamics. Revised Edition, W.A. Benjamin, New York, 251.

[4] Kaufman, M. (2002) Principles of Thermodynamics. Marcel Dekker, New York, 217, 218. http://dx.doi.org/10.1201/9780203909768

[5] Moore, W.J. (1972) Physical Chemistry. 4th Edition, Prentice-Hall, Englewood Cliffs, New Jersey, 538-539.

[6] Eyring, H., Henderson, D., Stover, B.J. and Eyring, E.M. (1982) Statistical Mechanics and Dynamics. 2nd Edition, John Wiley \& Sons, New York, 365.

[7] Levy, R.A. (1968) Principles of Solid State Physics. Academic Press, New York, 337-338.

[8] Stringer, J. (1967) An Introduction to the Electron Theory of Solids. Pergamon Press, Oxford, 224.

[9] Lehmann, G. and Ziesche, P. (1990) Electronic Properties of Metals. Elsivier, Amsterdam, 27-28.

[10] Eyring, H., Henderson, D., Stover, B.J. and Eyring, E.M. (1982) Statistical Mechanics and Dynamics. 2nd Edition, John Wiley \& Sons, New York, 527.

[11] Rickayzen, G. (1966) Proceedings of the Physical Society, 89, 129-134. http://dx.doi.org/10.1088/0370-1328/89/1/319

[12] Rockwood, A.L. (1984) Physical Review A, 30, 2843-2844. http://dx.doi.org/10.1103/PhysRevA.30.2843

[13] Rockwood, A.L. (2013) Electrochimica Acta, 107, 686-690. http://dx.doi.org/10.1016/j.electacta.2013.06.044

[14] Rockwood, A.L. (2013) Electrochimica Acta, 112, 706-711. http://dx.doi.org/10.1016/j.electacta.2013.08.190

[15] Tykodi, R.J. (2002) Thermodynamics of Systems in Nonequilbrium States. Thinker's Press, Davenport, Iowa, 304-306.

[16] Blatt, F.J., Schroeder, P.A., Foiles, C.L. and Greig, D. (1976) Thermoelectric Power of Metals. Plenum, New York, 164-167. http://dx.doi.org/10.1007/978-1-4613-4268-7

[17] Domenicali, C.A. (1954) Reviews of Modern Physics, 26, 237-275. http://dx.doi.org/10.1103/RevModPhys.26.237

[18] Rockwood, A.L. (2015) ChemPhysChem, 16, 1978-1991. http://dx.doi.org/10.1002/cphc.201500044

[19] Phillips, N.E. (1958) Physical Review, 114, 676-687. http://dx.doi.org/10.1103/PhysRev.114.676

[20] Averback, R.S. and Bass, J. (1971) Physical Review Letters, 26, 882-884. http://dx.doi.org/10.1103/PhysRevLett.26.882

[21] Hedi, L., Lundqvist, B.I. and Lundqvist, S. (1969) Journal of Research of the National Bureau of Standards A, Physics and Chemistry, 74A, 417-431. http://dx.doi.org/10.6028/jres.074A.032 